\title{
Effectivity of intravescical thermo-chemotherapy prophylaxis for patients with high recurrence and progression risk for non-muscle invasive bladder cancer
}

\author{
Ali Serdar Gözen ${ }^{1}$, Paolo Umari ${ }^{2}$, Walter Scheitlin ${ }^{1}$, Fuat Ernis Su ${ }^{3}$, Yigit Akin ${ }^{4}$, Jens Rassweiler ${ }^{1}$ \\ ${ }^{1}$ Department of Urology, SLK-Kliniken, University of Heidelberg, Heilbronn, Germany; \\ 2 Department of Urology, Cattinara Hospital, University of Trieste, Italy; \\ ${ }^{3}$ Department of Urology, Kanuni Sultan Süleyman Education and Research Hospital, Istanbul, Turkey; \\ ${ }^{4}$ Department of Urology, Harran University School of Medicine, Sanliurfa, Turkey.
}

\begin{abstract}
Summary BackgroundEAim: High grade non-muscle invasive bladder cancer (NMIBC) is common in urological practice. Most of these cancers are or become refractory to intravesical immunotherapy and chemotherapy. Here we evaluated the efficacy of combined local bladder hyperthermia and intravesical mitomycin-C (MMC) instillation in patients with high-risk recurrent NMIBC.

Materials and methods: Between February 2014 and December 2015, 18 patients with high risk NMIBC were enrolled. Patients were treated in an outpatient basis with 6 weekly induction sessions followed by monthly maintenance sessions with intravesical MMC in local hyperthermia with bladder wall thermo-chemotherapy (BWT) system (PelvixTT system, Elmedical Ltd., Hod Hasharon, Israel). The follow-up regimen included cystoscopy after the induction cycle and thereafter with regular intervals. Time to disease recurrence was defined as time from the first intravesical treatment to endoscopic or histological documentation of a new bladder tumour. Adverse events were recorded according to CTC 4.0 (Common Toxicity Criteria) score system.

Results: Mean age was 72 (32-87) years. 10 patients had multifocal disease, 9 had CIS, 6 had recurrent disease and 2 had highly recurrent disease ( $>3$ recurrences in a 24 months period). 6 patients underwent previous intravesical chemotherapy with MMC. The average number of maintenance sessions per patient was 7.6. After a mean follow-up of 433 days, 15 patients $(83.3 \%)$ were recurrence-free. 3 patients had tumour recurrence after a mean period of 248 days without progression. Side effects were limited to grade 1 in 2 patients and grade 2 in 1 patient.

Conclusions: BWT seems to be feasible and safe in high grade NMIBC. More studies are needed to identify the subgroup of patients who may benefit more from this treatment.
\end{abstract}

KEY WORDS: Bladder cancer; Mitomycin-C; Regional perfusion cancer chemotherapy.

Submitted 12 August 2016; Accepted 11 January 2017

\section{INTRODUCTION}

The non-muscle invasive bladder cancer (NMIBC) is the $4^{\text {th }}$ most common cancer in men and $12^{\text {th }}$ in women with a great impact on health care system in Europe (1). Nearly $80 \%$ of the patients with bladder cancer present with non- muscle invasive disease at the diagnosis (2). The mean characteristics of this cancer are high recurrence rates after transurethral resection and high risk of progression to muscle invasive disease (3). Although, intravesical chemotherapy and immunotherapy can significantly decrease the recurrence and progression rates, they still represent the main challenge in this field and more effective therapies are needed (4). Particularly challenging is the treatment of patients with high-risk tumours according to the European Organization for Research and Treatment of Cancer (EORTC) scoring system and those who are refractory to intravescical Bacillus CalmetteGuérin (BCG) instillation (1). Moreover, there has been a shortage of BCG in Europe.

Many approaches have been used to enhance the antitumor effects of intravesical chemotherapy (5). Several studies have shown promising results combining intravesical mitomycin-C (MMC) with hyperthermia. It was demonstrated that hyperthermia of the bladder wall improves MMC penetration into the deep bladder wall and can offer anticancer advantages over chemotherapy instillation alone (6).

The aim of this study was to evaluate the efficacy of combined intravesical MMC instillation and bladder hyperthermia in a selected group of patients with high-risk recurrent NMIBC in whom radical surgery was not an option or BCG treatment was contraindicated.

\section{Materials ANd Methods}

This was a retrospective evaluation of prospectively collected data. The Ethics Commitee of our institution approved the present study. All patients read, understood, and signed the consents forms. All procedures performed were in accordante with the Helsinky declaration or comparable ethical standards.

\section{Patient and data collection}

Between, February 2014 and December 2015, 24 patients with high risk NMIBC underwent adjuvant thermo-chemotherapy with MMC at our Department. The exclusion criteria were patients with muscle invasive 
bladder cancer, low risk bladder cancer, histology different than transitional, tumours involving the urethra or upper urinary tract, large bladder diverticulum $(>1 \mathrm{~cm})$, patients underwent previous partial cystectomy and impossibility to undergone catheterisation with a 20 French catheter. 18 patients were eligible for the analysis and were included in the study.

The other 6 patients did not meet the inclusion criteria, because 2 of them had incomplete data, 1 had muscle invasive disease, 1 had squamous tumour at histology, 1 had multiple tumours involving the urethra and 1 withdrew treatment at $3^{\text {rd }}$ instillation because of his personal decision. We used tumour node metastasis (TNM) classification of 2009 for defining levels of NMIBC in the study (7).

\section{Treatment}

Patients were treated on an outpatient basis with 6 weekly induction sessions followed by monthly maintenance sessions of intravesical MMC and bladder thermochemotherapy (BWT). The maintenance treatment was prosecuted for up to 1 year and patients with no recurrences underwent to an additional maintenance cycle every 3 months thereafter.

The Unithermia System (Pelvix TT system, Elmedical Ltd., Hod Hasharon, Israel) was used to heat the bladder wall, in all patients. This system is composed of a compact console with a peristaltic pump and a heat exchanger that delivers intravesical MMC through a 3-way silicon 20 French catheter. It allows to obtain a uniform hyperthermia all over the bladder by continuous flow of the heated MMC solution. According to previous studies, patients received $80 \mathrm{mg}$ of MMC in $50 \mathrm{ml} 0.9 \%$ saline, heated at $45^{\circ} \mathrm{C}$ in continuous circulation for 50 minutes (8) (Figure 1).

\section{Figure 1.}

The Unithermia System (Pelvix TT system, Elmedical Ltd., Hod Hasharon, Israel) used to heat the bladder wall. It is composed from a compact console with a peristaltic pump and a heat exchanger that delivers intravesical chemotherapy through a 3-way silicon 20 French catheter. The uniform hyperthermia at $45^{\circ} \mathrm{C}$ of $80 \mathrm{mg}$ MMC in 50 $\mathrm{ml} 0.9 \%$ saline solution is mantained for 50 minutes by the continuous flow.

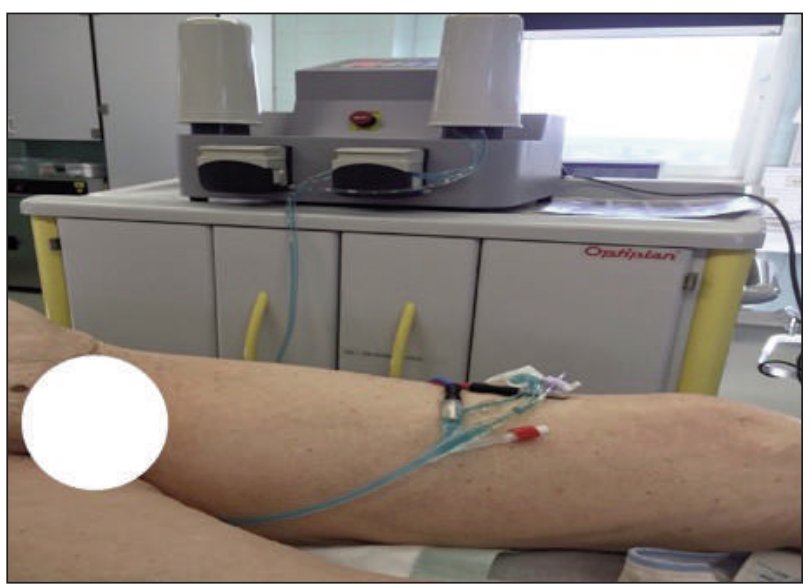

\section{Follow-up}

The follow-up regimen included cystoscopy after the induction cycle and after that every 3 months for a period of 2 years. Thereafter the follow-up was conducted every 6 months. All lesions that were detected by cystoscopy during follow-up underwent biopsy. The recurrence was evaluated by histological examination. Patients without tumour in the bladder and positive cytology underwent bladder mapping to exclude the presence of a carcinoma in-situ (CIS). The transitional cell carcinoma of the upper urinary tract was also rolled out with appropriate imaging in these cases. Time to disease recurrence was defined as the time from the first intravesical treatment to endoscopic or histological documentation of a new bladder tumour. Adverse events were recorded regarding the Common Toxicity Criteria (CTC) 4.0 score system (9).

\section{RESULTS}

\section{Description}

The mean age was 72 years (range 32-87). There were 16 male and 2 female patients. In total, 10 patients had multifocal disease, 9 had CIS, 7 had recurrent diseases and 2 of them had a highly recurrent disease ( $>3$ recurrences in a 24 months period). Both of last 2 patients had also a concurrent TlG3 disease. Stage before starting the BWT treatment was $\mathrm{Ta}$ in 5 patients, and $\mathrm{Tl}$ in 12 patients and all the patients had high grade (G2-3) disease. 6 patients have undergone previous intravesical therapy with MMC. The average number of maintenance sessions per patient was 7.6. All patients conducted the treatment during the induction and maintenance period with no significant side effects due to MMC and no physical complaints due to bladder hyperthermia. Table 1 summarizes patients' characteristics.

\section{Table 1.}

Patients characteristics.

\begin{tabular}{|lc|}
\hline Patients characteristics & \\
\hline Number of patients (total) & 24 \\
\hline Number of patients (included) & 18 \\
\hline Age mean (range) & $72(32-87)$ \\
\hline Gender & 16 \\
male & 2 \\
female & 7 \\
\hline Recurrence history & 2 \\
$\quad$ recurrent & 8 \\
highly recurrent & 10 \\
\hline Number of tumours before BWT & \\
unifocal & 5 \\
multifocal & 12 \\
\hline Tumor stage prior BWT & 9 \\
$\quad$ Ta & \\
T1 & 0 \\
CIS & 6 \\
\hline Prior intravescical therapy & 12 \\
BCG & \\
MMC & \\
none & \\
\hline BCG: Bacillus Calmette-Guérin; BWT: bladder wall thermo-chemotherapy; \\
MMC: Mitomycin C.
\end{tabular}




\section{Treatment results}

After a mean follow-up of 433 days, 15 patients (83.3\%) were recurrence-free. Besides, no patient had recurrence at the first cystoscopy after the induction cycle. 3 patients had tumour recurrence after a mean period of 248 days (range 191-339 days). However there was no disease progression, in fact staging and grading of these patients prior the treatment and at the time of reccurence were: TlG3+CIS, TaG2, CIS and TIG3, TaG1, CIS respectively. 2 of them had multifocal disease recurrence. None among the 2 patients with highly recurrent disease relapsed during a mean follow-up of 655 days. These results are summarized in Table 2 .

\section{Side effects}

Side effects according to CTC 4.0 were limited to grade 1 in 2 patients (skin allergy and haematuria) and grade 2 in 1 patient (pain and bladder spasm during induction treatment sessions treated with intravesical oxybutynin). Grade 1 complications were treated with oral analgesics and antiistaminics respectively.

\section{Table 2.}

\section{Review of the current series results.}

\begin{tabular}{|lc|}
\hline \multicolumn{2}{l}{ Parameters } \\
\hline Mean follow-up (days) & 433 \\
\hline Recurrence free rate & $15 / 18(83,3 \%)$ \\
\hline Mean recurrence time (days) & $248(191-339)$ \\
\hline Disease progression & $0 / 18(0 \%)$ \\
\hline Side effects (According to CTC) & \\
grade 1 & $2(11,1 \%)$ \\
grade 2 & $1(5,6 \%)$ \\
grade 3 & $0(0 \%)$ \\
grade 4 & $0(0 \%)$ \\
grade 5 & $0(0 \%)$ \\
\hline CTC: Common toxicity criteria. & \\
\hline
\end{tabular}

\section{Discussion}

The NMIBC is characterised by a high recurrence rate (30-85\%) after primary transurethral resection of the tumour (2). Thus, intravesical immunotherapy with BCG or MMC is used for preventing recurrence (1). It is well known that BCG can significantly reduce the disease progression and recurrence rate but at the same time can also produce serious side effects such as voiding problems, urinary infection, haematuria up to miliary tubercolosis $(10,11)$. In these cases only MMC can be used as intravescical therapy (12). On the other hand, since more than hundred years ago, many experiments have shown that cancer cells are more susceptible to hyperthermia than normal healthy cells (13). Local hyperthermia has a therapeutic potential for the treatment of many solid tumours, especially if used in combination with other treatments, such as radiation (Radio hyperthermia) and chemotherapy (Chemo hyperthermia) (14).

Lammers et al. reported a systematic review of 15 original articles and concluded that MMC+hyperthermia reduces the risk of NMIBC recurrence by $59 \%$ when compared to MMC alone despite a limited number of randomized tri- als in current literature (15). The bladder is particularly suitable for the application of local hyperthermia because its wall could be heated by the irrigation of warm fluid (15). Until now, several chemotherapeutics in various dosages and different regimens (adjuvant and neo-adjuvant) have been used in association with hyperthermia (16). Intravesical MMC was recently used for preventing the recurrence and the progression of NMIBC (17). It was demonstrated, that MMC associated with hyperthermia could have promising effects on a well selected subgroup of patients (18). It has been shown that hyperthermia interferes with DNA, RNA and protein synthesis of cancer cells. Moreover intravescical MMC can inactivate cell repair mechanisms by increasing tumour cell apoptosis (19). For these reasons intravescical MMC associated with thermotherapy may be used as prophylaxis for recurrence and progression in high grade NMIBC.

Heating of the bladder can be achieved, by radiofrequency, by magnetic nanoparticles in magnetic field or by circulation of externally heated fluid into the bladder (19). The most used systems are the Synergo system (SBTS $101 \pm 1$ System introduced by Colombo $R$ et al. in 1996) and the more recently Unithermia system $(20,21)$. The former is based on direct irradiation of the bladder lumen by a $915-\mathrm{MHz}$ intravesical microwave applicator and the latter comprises a console with a peristaltic pump and a heat exchanger that delivers intravesical chemotherapy through a 3-way silicon catheter (8). Another method was recently proposed and consists on regional hyperthermia therapy using an array of 70 to $120 \mathrm{MHz}$ antennas that are positioned around the patient, resulting in high homogenous temperatures of the target organ (22). We used the Unitherma system in the current study because of its easy applicability.

In our study thermo-chemotherapy with MMC provided promising results with low recurrence rate and no disease progression in patients with high grade NMIBC at a medium-term follow-up period. The overall rate of side effects was low and the acceptance of the treatment by the patient was high.

The main limitation of the current study is the small number of patients and therefore the impossibility to perform an accurate statistical analysis. The favorable factors are the only inclusion of patient with a high risk disease according to EORTC classification in whom radical surgery was not an option or BCG treatment was containdicated. Moreover all patients followed stricty the therapy plan and the follow-up schedule.

\section{Conclusions}

According to these data thermo-chemotherapy seems to be a feasible, safe and promising approach for prophylactic treatment in patients with high risk NMIBC. More studies are needed to identify the subgroup of patients who may benefit more from this treatment (ex. old patients with short life expectancy, those who are not candidates for anestesia due to high comorbidities or not suited for BCG therapy, those who refuse radical surgery and prefer conservative treatment to keep the bladder in situ). These preliminary results encourage further studies to define the limits and prospects of this regimen. 


\section{References}

1. http://uroweb.org/guideline/non-muscle-invasive-bladder-cancer/ Accessed June.16.2016.

2. Aldousari S, Kassouf W. Update on the management of non-muscle invasive bladder cancer. Can Urol Assoc J. 2010; 4:56-64.

3. Van Rhijn BW, Burger M, Lotan Y, et al. Recurrence and progression of disease in non-muscle-invasive bladder cancer: from epidemiology to treatment strategy. Eur Urol. 2009; 56:430-442.

4. Geijsen ED, de Reijke TM, Koning CC, et al. Combining Mitomycin C and Regional $70 \mathrm{MHz}$ Hyperthermia in Patients with Nonmuscle Invasive Bladder Cancer: A Pilot Study. J Urol. 2015; 194:1202-1208.

5. Arends TJ, Falke J, Lammers RJ, et al. Urinary cytokines in patients treated with intravesical mitomycin-C with and without hyperthermia. World J Urol. 2015; 33:1411-1417.

6. Maffezzini M, Campodonico F, Canepa G, et al. Intravesical mitomycin C combined with local microwave hyperthermia in nonmuscle-invasive bladder cancer with increased European Organization for Research and Treatment of Cancer (EORTC) score risk of recurrence and progression. Cancer Chemother Pharmacol 2014; 73:925-930.

7. UICC International Union Against Cancer. In: Sobin Lh, Gospodariwicz M, Wittekind C, editors. 7th ed. Oxford: WileyBlackwell TNM classification of malignant tumours. 2009; p.262265.

8. http://www.elmedical-group.com/home/doc.aspx?mCatID=13636. Accessed June.16.2016.

9. https://www.eortc.be/services/doc/ctc/CTCAE_4.03_2010-0614_QuickReference_5x7.pdf. Accessed June.16.2016.

10. Holz S, Sotorres Cabanillas JL, Legrand F, et al. Evaluation of adverse events caused by intravesical BCG instillations: Has the strain used a potential implication? Prog Urol. 2016; 26:73-78.

11. Rosati Y, Fabiani A, Taccari $T$, et al. Intravesical BCG therapy as cause of miliary pulmonary tuberculosis. Urologia. 2016; 82:49-53.

12. Porten SP, Leapman MS, Greene KL. Intravesical chemotherapy in non-muscle-invasive bladder cancer. Indian J Urol. 2015; 31:297- 303.
13. Edwards MJ. Apoptosis, the heat shock response, hyperthermia, birth defects, disease and cancer. Where are the common links? Cell Stress Chaperones. 1998; 3:213-220.

14. Hurwitz M, Stauffer P. Hyperthermia, radiationandchemotherapy: the role of heat in multidisciplinary cancer care. Semin Oncol. 2014; 41:714-729.

15. Lammers RJ, Witjes JA, Inman BA, et al. The role of a combined regimen with intravesical chemotherapy and hyperthermia in the management of non-muscle-invasive bladder cancer: a systematic review. Eur Urol. 2011; 60:81-93.

16. Colombo R. Combined treatment with local thermo-chemotherapy for non muscle invasive bladder cancer. The present role in the light of acquired data and preliminary cumulative clinical experiences. Arch Ital Urol Androl. 2008; 80:149-156.

17. Milla P, Fiorito C, Soria F, et al. Intravesicalthermo-chemotherapy based on conductive heat: a first pharmacokinetic study with mitomycin $C$ in superficial transitional cell carcinoma patients. Cancer Chemother Pharmacol. 2014; 73:503-509.

18. Owusu RA, Abern MR, Inman BA. Hyperthermia as adjunct to intravesical chemotherapy for bladder cancer. Biomed Res Int. 2013; 262313.

19. Slater SE, Patel P, Viney R, et al. The effects and effectiveness of electromotive drug administration and chemohyperthermia for treating non-muscle invasive bladder cancer. Ann R Coll Surg Engl. 2014; 96:415-419.

20. Kiss B, Schneider S, Thalmann GN, Roth B. Is thermochemotherapy with the Synergo system a viable treatment option in patients with recurrent non-muscle-invasive bladder cancer? Int J Urol. 2015; 22:158-162.

21. Soria F, Milla P, Fiorito $C$, et al. Efficacy and safety of a new device for intravesical thermochemotherapy in non-grade 3 BCG recurrent NMIBC: a phase I-II study. World J Urol. 2016; 34:189-195.

22. Geijsen ED, de Reijke TM, Koning CC, et al. Combining Mitomycin C and Regional $70 \mathrm{MHz}$ Hyperthermia in Patients with Nonmuscle Invasive Bladder Cancer: A Pilot Study. J Urol. 2015; 194:1202-1208.

\section{Correspondence}

Ali Serdar Gözen, MD, FEBU. Associate Professor of Urology (Corresponding Author) asgozen@yahoo.com

Walter Scheitlin, MD

Jens Rassweiler, MD

Department of Urology - SLK-Kliniken Heilbronn

Am Gesundbrunnen 20-26, D-74078 Heilbronn, Germany

Paolo Umari, MD

Department of Urology, Cattinara Hospital, University of Trieste

Trieste, Italy

Fuat Ernis Su, MD

Department of Urology, Kanuni Sultan Süleyman Education

and Research Hospital, Istanbul, Turkey

Yigit Akin, MD

Department of Urology, Harran University School of Medicine

Sanliurfa, Turkey 